\section{Kompetenznetz Demenzen - Früherkennung und Differenzialdiagnose}

Jens Wiltfang, Piotr Lewczuk, Johannes Miertschischk, Jana Svitek und Johannes Kornhuber

Klinik mit Poliklinik für Psychiatrie und Psychotherapie, Friedrich-Alexander-Universität ErlangenNürnberg

psychoneuro 2003; 29 (6): 290-293

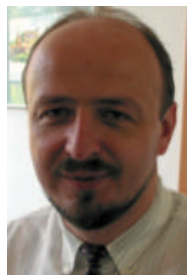

J. Wiltfang

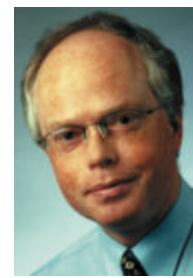

J. Kornhuber

Aufgrund der ausgeprägten Altersabhängigkeit von Demenzerkrankungen in Zusammenhang mit einer deutlich gestiegenen Lebenserwartung haben wir eine exponenzielle Zunahme der Prävalenz von Demenzerkrankungen zu erwarten. Zwischenzeitlich stehen erste Behandlungsansätze zur Verfügung, die bei frühzeitiger Intervention besonders effektiv sind. Bisher fehlt aber eine differenzierte Positivdiagnostik, um Demenzerkrankungen schon im Frühstadium zuverlässig zu diagnostizieren. Auch fehlen zuverlässige Verfahren, um bereits im Vorstadium der Demenzerkrankung, der leichten kognitiven Störung, vorherzusagen, welcher Patient voraussichtlich eine Demenzerkrankung entwickeln wird. Eine präklinische Diagnostik würde erlauben, diesen Patienten eine spezifische präventive Therapie anzubieten. Das Modul Frühund Differenzialdiagnostik (E1) des Kompetenznetzes Demenzen hat sich zur Aufgabe gemacht, innerhalb der drei Teilmodule Neuropsychologie, Neurochemische Demenzdiagnostik und Bildgebung die Positivdiagnostik früher Stadien von Demenzerkrankungen zu verbessern. Zusätzlich soll bei leichter kognitiver Störung die prädiktive Wertigkeit der diagnostischen Verfahren für die Vorhersage einer späteren Demenzerkrankung bestimmt werden. Ziel von Modul E1 ist damit die Etablierung einer zuverlässigen, validen und möglichst kostengünstigen Positivdiagnostik für die verbesserte Früh- und Differenzialdiagnostik von Demenzerkrankungen als Voraussetzung für ihre möglichst frühe Therapie.

\section{Modul Früh- und Differenzialdiagnose}

Die demographische Entwicklung unserer Altersstruktur bedingt in den kommenden Jahrzehnten eine starke Zunahme der Prävalenz von Demenzerkrankungen. Grund ist die starke Altersabhängigkeit von Demenzen gekoppelt mit einer höheren Lebenserwartung. So steigt die Prävalenz exponenziell mit zunehmendem Alter und erreicht bei Personen im Alter von 80 bis 89 Jah- ren etwa $10 \%$. Dabei entfällt mindestens die Hälfte der Erkrankungen auf die Alzheimer-Demenz. Zwischenzeitlich stehen verbesserte medikamentöse Behandlungsansätze der Alzheimer-Demenz zur Verfügung, wie beispielsweise die Behandlung mit Hemmstoffen der Azetylcholinesterase (AchE-Inhibitoren) und/oder Memantine. Diese Behandlungsansätze sind wahrscheinlich besonders effektiv, wenn sie frühzeitig eingesetzt werden.
Bisher fehlt aber eine differenzierte Positivdiagnostik, um Demenzerkrankungen auch schon im Frühstadium zuverlässig zu erkennen. Außerdem fehlen bisher zuverlässige Verfahren, mit denen bereits im Vorstadium der Demenzerkrankung, der leichten kognitiven Störung, vorher gesagt werden kann, welcher Patient voraussichtlich eine Demenzerkrankung entwickeln wird.

Ziel des Moduls Früh- und Differenzialdiagnostik (Leitung: J. Kornhuber, J. Wiltfang, Erlangen) ist es, mittels der Teilmodule Neuropsychologie, Neurochemische Demenzdiagnostik und Bildgebung die Positivdiagnostik früher Stadien von Demenzerkrankungen $\mathrm{zu}$ verbessern. Damit soll die Voraussetzung für eine möglichst frühe (präventive) Therapie geschaffen werden.

Um dieses Ziel zu erreichen, werden innerhalb von zwei Jahren durch die beteiligten 14 horizontalen Netzwerkpartner insgesamt 1000 Patienten mit Frühstadien demenzieller Erkrankungen und 1000 Patienten mit leichter kognitiver Störung eingeschlossen und verlaufsabhängig über drei Jahre (jährliche Kontrolle) untersucht. 
Teilmodul Psychopathologie, Demographie und Neuropsychologische Diagnostik

International gibt es bisher keine ausreichend validierten klinischen Instrumente für die Diagnostik früher Demenzstadien und keine verbindlichen Kriterien für die neuropsychologische Diagnostik der leichten kognitiven Störung. Auch ist für die neuropsychologischen Testverfahren nicht bekannt, ob durch ihren kombinierten Einsatz für den einzelnen Patienten der Übergang aus dem Stadium der leichten kognitiven Störung zur Demenz vorhergesagt werden kann. Diese Fragen sind Gegenstand des Teilmoduls Psychopathologie, Demographie und Neuropsychologische Diagnostik (Leitung: A. Kurz, TU München; F. Reischies, FU Berlin). Hier werden auch mehrere neuropsychologische Testverfahren vergleichend eingesetzt, um bestimmte Aspekte der Krankheitsprogression möglichst zuverlässig abzubilden, und therapeutische Effekte ausreichend sensitiv nachweisen zu können. Durch dieses Teilmodul wurden auch die essenziellen klinischen und laborchemischen Parameter festgelegt, die für eine „state-of-the-art“ Demenzdiagnostik unverzichtbar sind. Diese Parametergruppe schließt dabei die klinischen Informationen mit ein, die auch in-

nerhalb des Kompetenznetzwerkes Parkinson und des „BrainNet“ erhoben werden. Durch regelmäßige

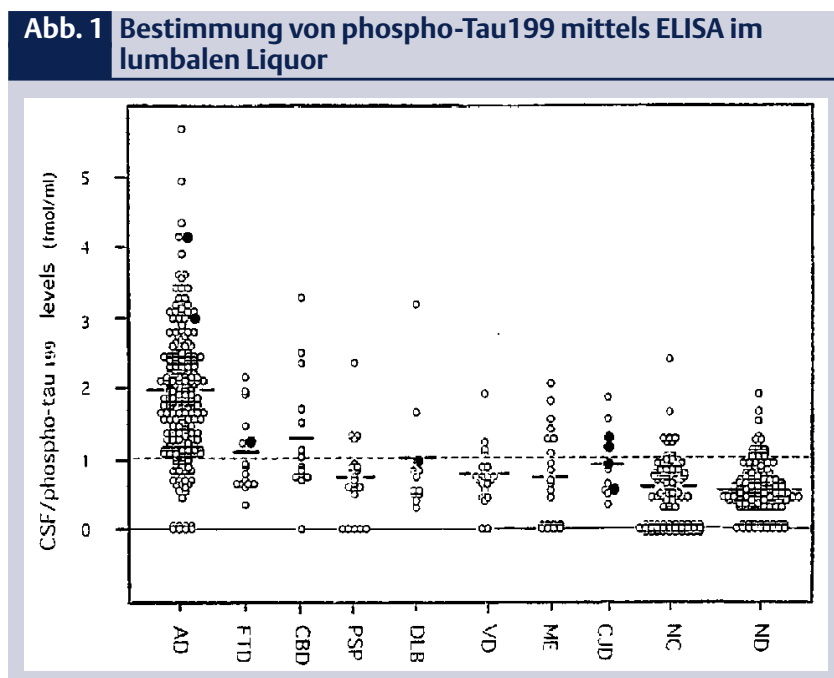

Für die Differenzierung AD versus alle anderen Erkrankungen errechnet sich eine Spezifität und Sensitivität von jeweils 85\%. AD: Alzheimer-Demenz, n=236; FTD: Frontotemporale Demenzen, n=16; CBD: Corticobasale Degeneration, $n=15$; PSP: Progressive supranukleäre Lähmung, n=21; DLB: Lewy-Körper-Demenz, n=13; VD: Vaskuläre Demenzen, n=23; ME: Meningoenzephalitis, n=18; CJD: Creutzfeldt-Jakob Demenz, n=10; NC: gesunde Kontrollen, n=95; ND: Neuropsychiatrische Kontrollpatienten, $n=122$. Modifiziert nach Itoh et al., Annals of Neurology, 2001.

\section{Abb. 2 Biochip-basierte Massenspektroskopie der Aß-Peptidspezies im lumbalen Liquor}

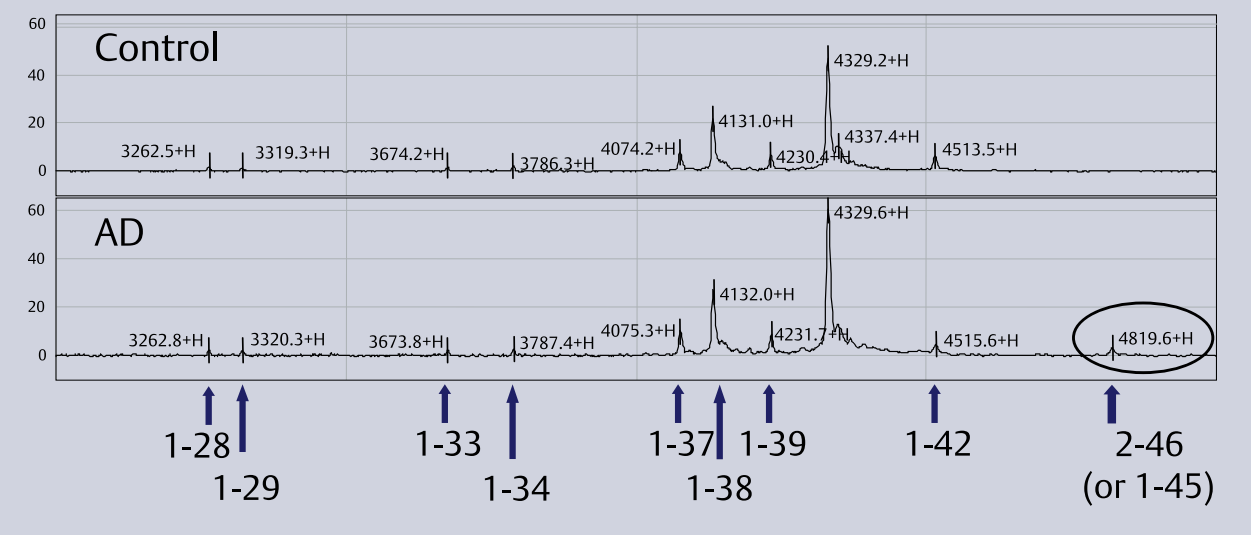

Der Biochip wurde von uns mit einem monoklonalem anti-Aß-Peptidantikörper kovalent beschichtet. Die Messung erfolgte mittels SELDI-TOF-MS ( „surface enhanced laser desorption ionisation time of flight mass spectroscopy“). Neben der bekannten Reduktion von $A \beta_{1-42}$ bei $A D$ kann das Vorkommen von $A \beta_{1-37 / 38 / 39}$ und weiterer carboxyterminal-verkürzter Aß-Peptide bestätigt werden. Zusätzlich gelingt uns erstmals der Nachweis eines weiteren Aß-Peptids (Mr 4819.6), das nur im Liquor bei AD gefunden wurde. Modifiziert nach Lewczuk et al., Rapid Communications in Mass Spectroscopy, 2003. 
Schulungen der Kollegen an den beteiligten 14 universitären Zentren („Rater Training“) wird eine standardisierte und reliable Erhebung der Daten gewährleistet. Diese Maßnahmen erlauben eine Qualitätssicherung der klinischen und neuropsychologischen Diagnostik auf hohem Niveau und sind die unverzichtbare Voraussetzung, um nachgeschaltete differenzierte Forschungsfragestellungen multizentrisch sinnvoll bearbeiten zu können. Die Parametergruppen, diagnostischen Verfahren und Untersuchungsabläufe werden zurzeit in einem Handbuch dokumentiert, das später interessierten Kollegen zur Verfügung gestellt werden kann.

\section{Teilmodul Neurochemische Demenzdiagnostik}

Die neurochemische Demenzdiagnostik hat sich zwischenzeitlich von einer traditionellen Ausschlussdiagnostik zur Positivdiagnostik gewandelt. Ausreichend validierte Verfahren stehen bisher nur für neurochemische Demenzmarker im lumbalen Liquor zur Verfügung, obwohl sich erste Ansätze für Blutassays der Alzheimer-Demenz abzeichnen. Innerhalb des Teilmoduls Neurochemische Demenzdiagnostik (Leitung: J. Wiltfang, J. Kornhuber, Erlangen) wird zwischen einem Basismodul und einem Forschungsmodul unterschieden. Das Basismodul untersucht ausschließlich neurochemische Demenzmarker, deren Assays technisch eine Hochdurchsatzanalytik erlauben und deren diagnostische Validität in unabhängigen multizentrischen Studien mit Einschluss einer ausreichend hohen Fallzahl von Demenzerkrankungen unterschiedlicher Ätiologie gezeigt wurde. Teil dieser Kerngruppe neurochemischer Demenzmarker sind die beta-Amyloidpeptide (Aß-Peptide) 1-40, 1-42 und zusätzlich das Gesamt-Tauprotein, sowie phosphorylierte Tauproteine (Abb. 1). Begleitend wird eine ApoE-Genotypisierung innerhalb des Moduls Genetik und Epidemiologie (Leitung: W. Maier, Bonn; M.C. Angermeyer, Leipzig) durchgeführt. Dies dient unter anderem dazu, zukünftig ApoE $\epsilon 4$ spezifische Referenzbereiche für $A ß_{1-42}$ aufzubauen, da die Liquorkonzentration dieses Aß-Peptids unabhängig vom Vorliegen einer Alzheimer-Demenz invers mit der Gendosis von ApoE $\epsilon 4$ korreliert ist. Inhaltlich liegt ein Schwerpunkt auf der verbesserten Frühdiagnostik demenzieller Erkrankungen. Da bestimmte neurochemische Demenzmarker wohl primär mit der Pathobiochemie der Alzheimer-Krankheit und erst sekundär mit der später einsetzenden Alzheimer-Demenz assoziiert sind, ist zu erwarten, dass die neurochemische Demenzdiagnostik auch für eine präklinische Diagnostik der Alzheimer-Demenz eingesetzt werden kann. Erste Ergebnisse zu Veränderungen von $A ß-$ Peptiden und Phospho-Tauproteinen

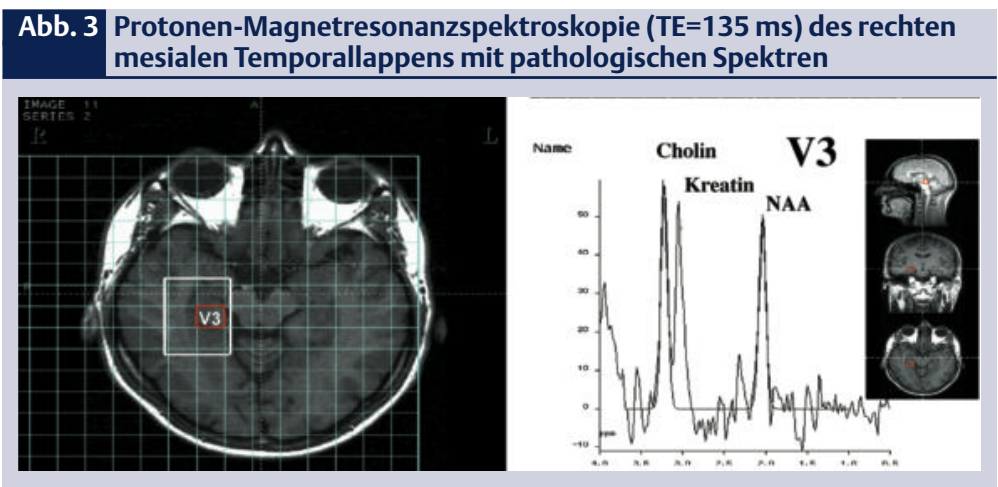

Relativ zu Cholin findet sich eine Abnahme des neuronalen Stoffwechselproduktes N-Azetylaspartat (die Aufnahme wurde von Dr. T. Hammen, Friedrich-Alexander-Universität Erlangen-Nürnberg zur Verfügung gestellt

im lumbalen Liquor bei Patienten mit leichter kognitiver Störung, die verlaufsabhängig untersucht wurden, bestätigen diese Annahme. Ein weiterer Schwerpunkt des Basismoduls liegt in der verbesserten Standardisierung der prä-analytischen Probenbehandlung. Hier wurde erstmals ein Monitoring der Probengewinnung aufgebaut, wobei der Prozess von der Probenentnahme bis zum Abschluss der Vorbehandlung verbindlich dokumentiert wird. Die neurochemische Demenzdiagnostik wird vom Referenzlabor in Erlangen koordiniert. Zwei weitere Referenzlabore sind an den Psychiatrischen Abteilungen der Universitäten in Göttingen und München (LMU) etabliert. Durch die Schaffung dieser drei Referenzlabore sind Ringversuche möglich, die für die Qualitätssicherung und Validierung neuer Assays unerlässlich sind.

Innerhalb des Forschungsmoduls der Neurochemischen Demenzdiagnostik sollen über den Einsatz neuer methodischer Verfahren aus dem Bereich „clinical proteomics“ (Abb. 2) neuartige neurochemische Demenzmarker in Liquor, Plasma und Serum identifiziert werden. Ein weiterer Forschungsschwerpunkt besteht in der Entwicklung so genannter „Multiplex Assays“, die die gleichzeitige Bestimmung mehrerer Demenzmarker im selben Probenvolumen zulassen (Demenzbiochip).

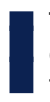

Teilmodul Bildgebung: craniales NMR, NMR-Volumetrie, NMR-Spektroskopie

Das dritte Standbein einer differenzierten Demenzdiagnostik sind bildgebende Verfahren. Der Bereich Bildgebung (Leitung: R. Heun, Bonn; H. Hampel, München) teilt sich in die morphometrischen kernspintomographischen Untersuchungen mit der Referenzzentrale an der LMU München (Leitung: H. Hampel) und die spektroskopischen kernspintomographischen Untersuchungen, die von der Psychiatrischen Klinik in Bonn koordiniert werden (Leitung: R. Heun). Bei allen Patienten wird ein Routine-NMR durchgeführt, das nach standardisierten Kriterien ausgewertet wird. Zusätzlich erfolgt bei einem Teil der rekrutierten Patienten 
eine Hippokampus-Volumetrie, die bei verlaufsabhängiger Untersuchung differenzialdiagnostisch besonders wertvoll ist und auch für die objektive Beurteilung der Effektivität der medikamentösen Behandlung eingesetzt wird. Die spektroskopischen Untersuchungen mit nicht-invasiver Bestimmung zentralnervöser Stoffwechselprodukte, wie N-Azetylaspartat, Myo-Inositol, oder Cholin, kann in sieben der beteiligten 14 Zentren durchgeführt werden (Abb. 3). Neben Einsatz der Methodik für die Abbildung von Therapieeffekten soll hier untersucht werden, inwieweit durch die spektroskopischen Untersuchungen die Frühdiagnostik demenzieller Erkrankungen verbessert werden kann. Bei Patienten mit leichter kognitiver Störung soll auch festgestellt werden, inwieweit das Auftreten einer späteren Demenzerkrankung vorhergesagt werden kann. Ein methodischer Schwerpunkt dieses Teilmoduls besteht in der verbesserten Standardisierung der bildgebenden Verfahren, wobei sowohl die Datenerhebung als auch die Datenauswertung zwischen den Zentren harmonisiert werden. Um die Vergleichbarkeit der Messungen zwischen den beteiligten Zentren überprüfen zu können werden so genannte „Phantommessungen“ eingesetzt. Diese methodische Pionierarbeit erscheint unverzichtbar, um auch bei einer größeren multizentrischen Studie Veränderungen im Krankheitsverlauf und Therapieeffekte möglichst sensitiv abbilden zu können.

\section{Summary}

Due to the age dependency of dementias and the increase in life expectancy we have to face an exponential increase in the prevalence of dementias. Meanwhile first therapies of dementias have become available, which are most effective, if treatment starts early. However, still a differentiated positive diagnosis for early dementias is missing. Moreover, there is a need for reliable diagnostic tools, which allow to predict the development of a subsequent dementia already in its preclinical stage, that is mild cognitive impairment. A preclinical diagnosis would allow to offer the patient a preventive therapeutic intervention. The module early and differential diagnosis (E1) of the competence network dementias applies the three submodules neuropsychology, neurochemical dementia diagnostics, and imaging to improve the early diagnosis of dementias. Moreover, diagnostics tools are identified, which allow the prediction of a subsequent dementia already at the preclinical stage of mild cognitive impairment. Accordingly, the main scope of E1 is to set up a reliable, valid, and economic positive diagnosis for the improved early and differential diagnosis of dementias. This will support their early therapy.

\section{Key Words}

Alzheimer's dementia - neurochemical dementia markers neuroimaging-neuropsychological tests

\footnotetext{
Korrespondenzadresse:

Prof. Dr. Jens Wiltfang

Klinik mit Poliklinik für Psychiatrie

und Psychotherapie

Friedrich-Alexander-Universität

Erlangen-Nürnberg

Schwabachanlage 6

91054 Erlangen
}

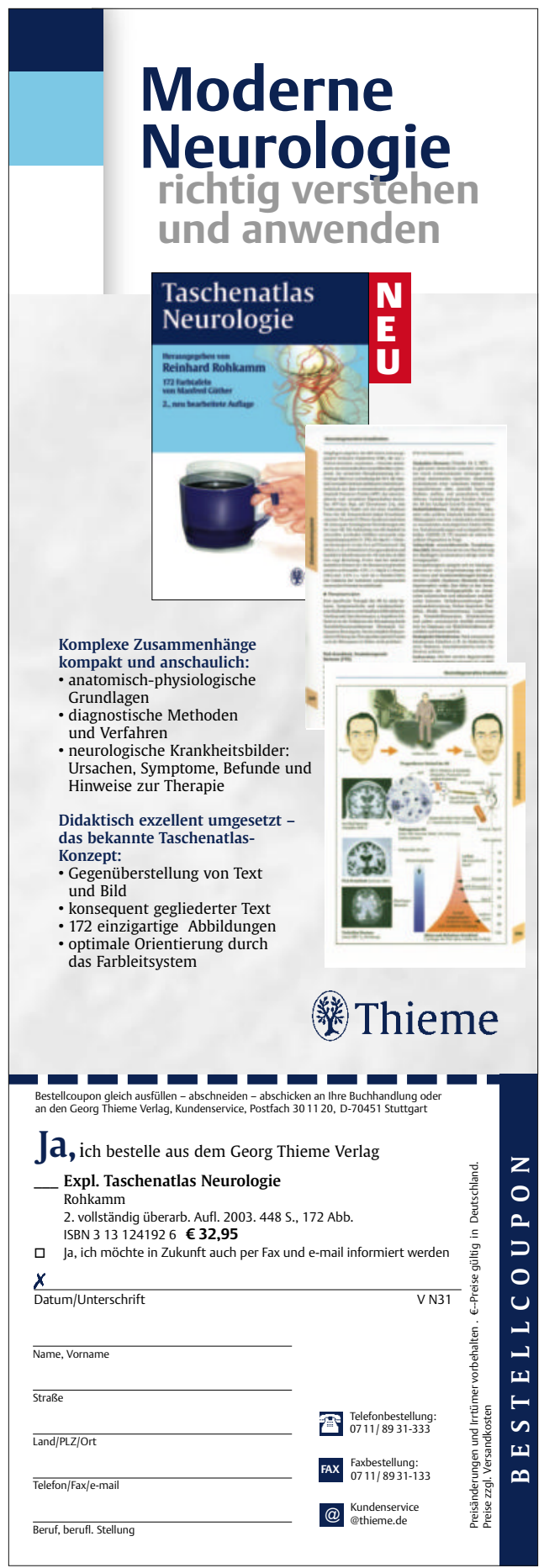

